\title{
Testing process performance based on the yield: an application to the liquid-crystal display module
}

\author{
Jann-Pygn Chen ${ }^{\mathrm{a}, *}$, W.L. Pearn ${ }^{\mathrm{b}}$ \\ ${ }^{a}$ Department of Industrial Engineering and Management, National Chin-Yi Institute of Technology, No. 35, Lane 215, Section 1, \\ Chung Shan Road, Taiping, Taichung, 411 Taiwan, ROC \\ ${ }^{\mathrm{b}}$ Department of Industrial Engineering and Management, National Chiao Tung University, Taiwan, ROC
}

Received 17 January 2002; received in revised form 18 March 2002

\begin{abstract}
Process capability indices have been introduced to provide numerical measures on whether a manufacturing process is capable of reproducing items meeting the specifications predetermined by the product designers or the consumers. Process yield is one of the most common criteria used in the manufacturing industry for measuring process performance. The formula $S_{\mathrm{pk}}$ has been proposed to calculate the process yield for normal processes. The formula $S_{\mathrm{pk}}$ provides an exact measure on the process yield. Unfortunately, the statistical properties of the estimated $\hat{S}_{\mathrm{pk}}$ are mathematically intractable. In this paper, we apply the bootstrap simulation method to construct the lower confidence bound of $S_{\mathrm{pk}}$. We then present a real-world application to the liquid-crystal display module process, to illustrate how we may apply the formula $S_{\mathrm{pk}}$ to actual data collected from the factories.
\end{abstract}

(C) 2002 Elsevier Science Ltd. All rights reserved.

\section{Introduction}

Process capability indices (PCIs) have been proposed to the manufacturing industry to provide numerical measures on whether a process is capable of producing product items within the specification limits predetermined by the product designers or the consumers. The PCIs were first applied to the automatic industry in Japan and America. In a purchasing contract, a minimum value of the PCI is usually specified. If the prescribed minimum value of the PCI fails to be met, the process is determined to be incapable. Otherwise, the process will be determined to be capable.

Process yield has longtime been the most common and standard criteria used in various manufacturing industries to characterize the process performance. Process yield is currently defined as the percentage of the processed product units passing the inspections. That is, the product characteristic must fall within the manu-

\footnotetext{
*Corresponding author. Tel.: +886-4-392-4505x7642; fax: +886-4-393-4620.

E-mail address: chen504@chinyi.ncit.edu.tw (J.-P. Chen).
}

facturing tolerance. For product units rejected during the inspection (nonconforming), additional costs would be incurred to the factory for scrapping or reworking the product. All passed product units are treated equally and accepted by the producer, which requires no additional cost to the factory.

For processes involving two-sided manufacturing specifications, the process yield can be calculated as Yield $=F(\mathrm{USL})-F(\mathrm{LSL})$, where USL and LSL are the upper and the lower specification limits, respectively, and $F(x)$ is the cumulative distribution function of the process characteristic. If the process characteristic is normally distributed, then the process yield can be expressed as Yield $=\Phi[(\mathrm{USL}-\mu) / \sigma]-\Phi[(\mathrm{LSL}-\mu) / \sigma]$, where the parameter $\mu$ is the process mean, parameter $\sigma$ is the process standard deviation, and $\Phi(x)$ is the cumulative distribution function of the standard normal distribution $N(0,1)$. Based on the above expression of the process yield, Boyles [1] considered the index $S_{\mathrm{pk}}$ to calculate the yield for normal processes, defined as

$$
S_{\mathrm{pk}}=\frac{1}{3} \Phi^{-1}\left\{\frac{1}{2} \Phi\left(\frac{\mathrm{USL}-\mu}{\sigma}\right)+\frac{1}{2} \Phi\left(\frac{\mu-\mathrm{LSL}}{\sigma}\right)\right\} .
$$




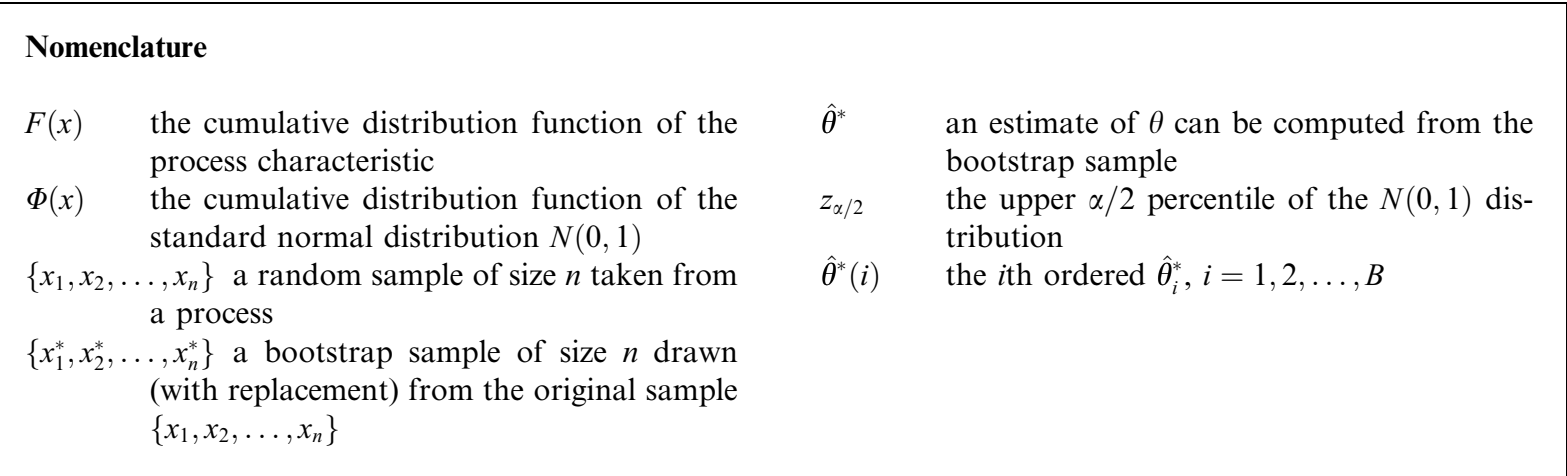

The index $S_{\text {pk }}$ establishes the relationship between the manufacturing specifications and the actual process performance, which provides an exact measure on the process yield. If $S_{\mathrm{pk}}=c$, then the process yield can be expressed as Yield $=2 \Phi(3 c)-1$. The fraction of nonconforming is defined as the percentage of product items fall outside the specification limits, which can be represented as $1-$ Yield $=2\left(1-\Phi\left(3 S_{\mathrm{pk}}\right)\right)$. Obviously, there is a one-to-one correspondence between $S_{\mathrm{pk}}$ and the process yield. Thus, $S_{\mathrm{pk}}$ provides an exact measure of the process yield. Note that other existing methods only provide approximate (instead of exact) yield measures. Table 1 displays the fraction of nonconforming (in ppm) as a function of the index $S_{\mathrm{pk}}$, (calculated by SAS computer programs).

\section{Estimating the measure $S_{\mathrm{pk}}$}

A natural estimator of the index $S_{\mathrm{pk}}$ may be obtained by replacing the unknown process mean $\mu$ by the sample mean $\bar{X}=\left(X_{1}+X_{2}+\cdots+X_{n}\right) / n$, and the unknown process standard deviation $\sigma$ by the sample standard deviation, $S=\left[\sum\left(X_{i}-\bar{X}\right)^{2} /(n-1)\right]^{1 / 2}$, respectively, which may be obtained from a stable (statistically in control) process. Thus, the natural estimator $\hat{S}_{\mathrm{pk}}$ may be expressed as

$\hat{S}_{\mathrm{pk}}=\frac{1}{3} \Phi^{-1}\left\{\frac{1}{2} \Phi\left(\frac{\mathrm{USL}-\bar{X}}{S}\right)+\frac{1}{2} \Phi\left(\frac{\bar{X}-\mathrm{LSL}}{S}\right)\right\}$.

Unfortunately, the exact distribution of $\hat{S}_{\mathrm{pk}}$ is mathematically intractable. Lee et al. [2] derived an approximate distribution of the estimator $\hat{S}_{\mathrm{pk}}$ using Taylor expansion technique. They showed that the estimator $\hat{S}_{\mathrm{pk}}$ is approximately distributed as the normal distribu- tion. The calculation of the approximation is, however, rather messy, and cumbersome to deal with. Further, the accuracy of the approximation has not been investigated. Thus, the approximation would not be practically useful until the difficulty is overcome. For practical purpose, in this paper we use the bootstrap resampling technique to find the lower confidence bound on $S_{\mathrm{pk}}$, so that practitioners/engineers can use them to perform quality testing and determine whether their process meets the preset quality requirement. We also present a case study on a liquid-crystal display module (LCM) manufacturing process to illustrate how the bootstrap lower confidence limit of $S_{\mathrm{pk}}$ may be applied to actual process data collected from the factory.

\section{The bootstrap methodology}

Efron $[3,4]$ introduced a nonparametric, computational intensive but effective estimation method called "the bootstrap", which is a data based simulation technique for statistical inference. In particular, one can use the nonparametric bootstrap method to estimate the sampling distribution of a statistic, while assuming only that the sample is a representative of the population from which it is drawn, and that the observations are independent and identically distributed. The merit, in its simplest form, is that the nonparametric bootstrap does not rely on any distributional assumptions about the underlying population.

Suppose the set of observations $\left\{x_{1}, x_{2}, \ldots, x_{n}\right\}$ is a random sample of size $n$ taken from a process. A bootstrap sample, $\left\{x_{1}^{*}, x_{2}^{*}, \ldots, x_{n}^{*}\right\}$, is a sample of size $n$ drawn (with replacement) from the original sample $\left\{x_{1}, x_{2}, \ldots, x_{n}\right\}$. Hence, there are a total of $n^{n}$ possible

Table 1

Various $S_{\mathrm{pk}}$ values and the fraction of nonconformities (in ppm)

\begin{tabular}{|c|c|c|c|c|c|c|c|c|c|c|c|c|c|c|c|c|}
\hline$S_{\mathrm{pk}}$ & 0.25 & 0.50 & 0.60 & 0.70 & 0.80 & 0.90 & 1.00 & 1.10 & 1.20 & 1.30 & 1.40 & 1.50 & 1.60 & 1.70 & 1.80 & 2.00 \\
\hline ppm & 453255 & 133614 & 71861 & 35729 & 16395 & 6934 & 2700 & 967 & 318 & 96 & 27 & 7 & 2 & 0.34 & 0.07 & 0 \\
\hline
\end{tabular}


resamples. Thus, the bootstrap sampling is equivalent to sampling with replacement from the empirical distribution function, and the bootstrap distribution is an estimator of the distribution of $\hat{S}_{\mathrm{pk}}$. In practice, usually only a small number of random samples out of the $n^{n}$ possible resamples is drawn, the estimate is calculated for each of these, and the subsequent empirical distribution is referred to as the statistic's bootstrap distribution. Efron and Tibshirani [5] indicated that a rough minimum of 1000 bootstrap resamples is usually sufficient for computing confidence interval estimates with reasonably accuracy.

Suppose the random variable $X$ measures process performance with respective to certain quality characteristic. The distribution of $X$ is generally unknown. Suppose we wish to estimate some parameter, $\theta$, that characterizes the performance of the process. An estimate of $\theta$ can be computed from the bootstrap sample, denoted as $\hat{\theta}^{*}$, which is called the bootstrap estimate. The resampling procedure can be performed repeatedly to obtain a certain number of bootstrap samples, for example, $B$ times. Then, the $B$ bootstrap estimates $\hat{\theta}_{1}^{*}, \hat{\theta}_{2}^{*}, \ldots, \hat{\theta}_{B}^{*}$, can be computed from the resamples. Research papers discussing the bootstrap methods include Efron and Gong [6], Gunter [7,8], Mooney and Duval [9], Young [10], and many others. In particular, Efron and Tibshirani [5] developed three types of bootstrap confidence interval. Those include the standard bootstrap (SB) confidence interval, the percentile bootstrap (PB) confidence interval, and the biased-corrected percentile bootstrap (BCPB) confidence interval, which are defined as follows:

(1) Standard bootstrap: From the $B$ bootstrap estimates $\left\{\hat{\theta}_{i}^{*}\right\}, i=1,2, \ldots, B$, we calculate the sample average, and the sample standard deviation as

$\bar{\theta}^{*}=\frac{1}{B} \sum_{i=1}^{B} \hat{\theta}_{i}^{*}$

$S_{\hat{\theta}}^{*}=\sqrt{\frac{1}{B-1} \sum_{i=1}^{B}\left(\hat{\theta}_{i}^{*}-\bar{\theta}^{*}\right)^{2}}$.

If the distribution of the estimate $\hat{\theta}$ is approximately normal, the $(1-\alpha) 100 \%$ level SB confidence interval for $\theta$ is $\hat{\theta}^{*} \pm z_{\alpha / 2} S_{\hat{\theta}}^{*}$, where $z_{\alpha / 2}$ is the upper $\alpha / 2$ percentile of the $N(0,1)$ distribution.

(2) Percentile bootstrap: From the ordered collection of $\left\{\hat{\theta}_{i}^{*}\right\}, i=1,2, \ldots, B$, the $\alpha / 2$ percentage and the $(1-\alpha / 2)$ percentage points are used to obtain the $(1-\alpha) 100 \%$ level PB confidence interval for $\theta$, where $\hat{\theta}^{*}(i)$ is the $i$ th ordered $\hat{\theta}_{i}^{*}, i=1,2, \ldots, B$.

$\left[\hat{\theta}^{*}(\alpha / 2 \times B), \hat{\theta}^{*}((1-\alpha / 2) \times B)\right]$.
(3) Biased-corrected PB: The bootstrap distribution obtained using only a sample of the complete bootstrap distribution may be shifted higher or lower than would be expected (i.e, a biased distribution). Thus, a modified method is developed to correct for this potential bias (see Efron [4] for a complete justification of this method). First, we use the (ordered) distribution of $\hat{\theta}_{i}^{*}$ to calculate the probability $p_{0}=\operatorname{Pr}\left[\hat{\theta}^{*}(i) \leqslant \hat{\theta}\right], i=1$, $2, \ldots, B$, where $\hat{\theta}$ is the estimated value of $\theta$ using a random sample $\left\{x_{1}, x_{2}, \ldots, x_{n}\right\}$. Second, we calculate the percentile point $z_{0}=\Phi^{-1}\left(p_{0}\right)$, and $P_{\mathrm{L}}=\Phi\left(2 z_{0}-z_{\alpha / 2}\right)$, $P_{\mathrm{U}}=\Phi\left(2 z_{0}+z_{\alpha / 2}\right)$, where $\Phi($.$) is the standard normal$ cumulative distribution function. Finally, the BCPB confidence interval is found as $\left[\hat{\theta}^{*}\left(P_{\mathrm{L}} \mathrm{B}\right), \hat{\theta}^{*}\left(P_{\mathrm{U}} \mathrm{B}\right)\right]$.

Franklin and Wasserman [11] investigated the lower confidence bounds for the capability indices, $C_{\mathrm{p}}, C_{\mathrm{pk}}$ and $C_{\mathrm{pm}}$ using the three bootstrap methods. Some simulations were conducted, and a comparison was made among the three bootstrap methods based on the parametric estimates. The simulation results indicate that for normal processes the bootstrap confidence limits perform equally well (see Chou et al. [12], Bissell [13], and Boyles [14]). And for nonnormal processes the bootstrap estimates performed significantly better than other methods. Franklin and Wasserman [11] also found that in all cases investigated the SB method performs better than the PB and the BCPB methods. Pearn and Chen [15] also applied the three bootstrap methods to find the lower confidence bounds of the yield measure $S_{\mathrm{pk}}$, and compared their performance based on the coverage percentage. Their results showed that the SB performs better than the other two methods. In particular, as the sample size $n$ exceeds 45 , the coverage percentage of the $95 \%$ lower confidence bound for the SB method, is greater than $90 \%$.

\section{LCM manufacturing process}

To illustrate how the bootstrap lower confidence bound of $S_{\text {pk }}$ may be established and applied to the actual data collected from the factories, we present a case study on the liquid-crystal module manufacturing process. The case we investigated was taken from a manufacturing factory located on the Science-Based Industrial Park, Taiwan, making the LCMs. The LCM is one of the key components used in many high-tech electronic commercial devices, such as the cellular phone, the PDA (personal digital assistant), the pocket calculator, digital watch, automobile accessory visual displays, and many others. Three key components make the LCM functions properly. Those include the liquid-crystal display, the back lighting, and the peripheral (interface) system. A typical assembly drawing for the LCM product is depicted in Fig. 1. 

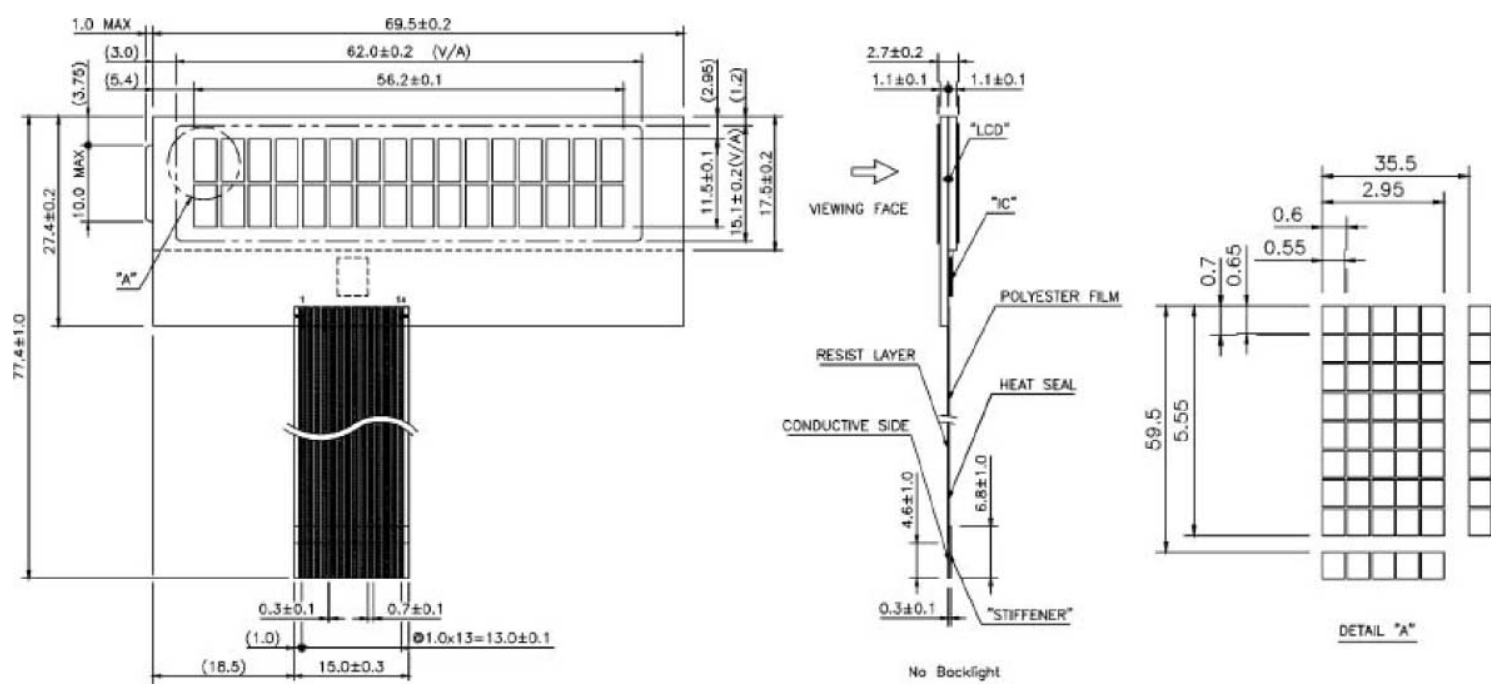

Fig. 1. The assembly drawing of the LCM product.

The mounting technology for the chip on glass (COG) makes the exposed particle overturned, with the side of circuits facing downward. Then, the electricity conduction is joined between the IC and the panel of the liquid-crystal display through the mounting material. Currently, the mounting technology of the COG is the best manufacturing technology for the LCM in terms of the mounting density. It is important to note that different mounting material requires different mounting technology of the COG.

In the factory, the manufacturing process control flow-chart of the COG is illustrated in Fig. 2. For the main bonding process, the bonding precision is an es- sential process parameter we focused on in our study. We investigated a particular model of the LCM product with the upper and the lower specification limits set to $\mathrm{USL}=15 \mu \mathrm{m}, \mathrm{LSL}=-15 \mu \mathrm{m}$, and the target value is set to $T=0$. If the characteristic data does not fall within the tolerance (LSL, USL), the lifetime or reliability of the LCM will be discounted. To ensure the production quality, and to satisfy the customers' requirement, the company has set the yield index $S_{\mathrm{pk}} \geqslant$ 1.50 , which implies that no more than $7 \mathrm{ppm}$ fraction of nonconforming for the product. If the capability requirement fails to be met, the LCM product would be seriously affected on its reliability or lifetime.

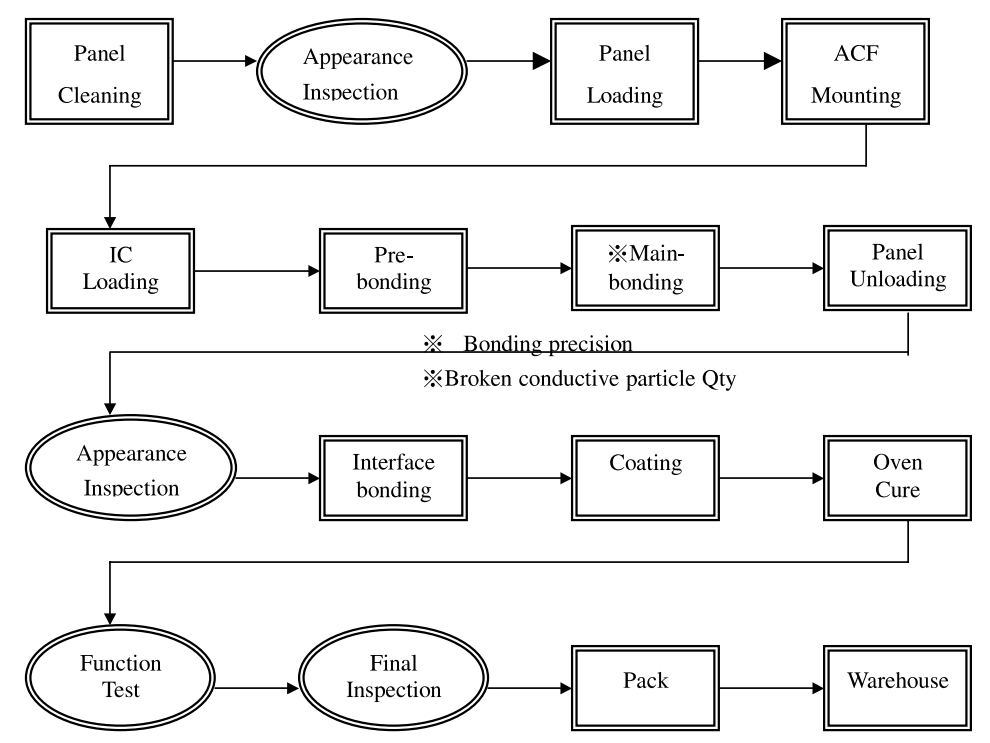

Fig. 2. Flow-chart of the COG manufacturing process. 
Table 2

The collected sample data with 64 observations (unit: $\mu \mathrm{m}$ )

\begin{tabular}{lrrr}
\hline-0.98 & 4.63 & 0.78 & -1.67 \\
2.34 & -1.97 & -0.07 & -2.32 \\
2.18 & 3.79 & 0.35 & -1.20 \\
3.13 & -5.58 & -1.91 & -1.18 \\
-0.66 & -3.16 & 5.01 & -1.42 \\
2.20 & 2.20 & 2.16 & -2.87 \\
-0.31 & 0.83 & -0.86 & -2.04 \\
1.24 & 5.83 & 0.50 & -0.81 \\
3.07 & -1.42 & -2.42 & -4.75 \\
1.02 & -6.51 & 1.34 & 1.06 \\
0.10 & 5.02 & -0.76 & -4.84 \\
1.28 & 2.19 & 0.16 & -2.66 \\
-0.87 & -3.52 & -0.05 & 1.11 \\
1.47 & 0.28 & -1.02 & -9.23 \\
-2.01 & -2.30 & 4.26 & 3.41 \\
3.34 & 2.85 & -2.43 & -1.84 \\
\hline
\end{tabular}

\subsection{The lower confidence bound}

In order to evaluate the process capability based on the $95 \%$ lower confidence limit using the SB method. According to the process control plan of the COG, the random sample data in consecutive two day are collected, which are displayed in Table 2. The sample observations are obtained through the inspection, using microscope by visual, which were collected eight pieces per every $2 \mathrm{~h}$. These observations were justified taken from a stable process, and the characteristic distribution is shown to be approximate normal.

Then, $B=10000$ bootstrap resamples (each is of size 64) are drawn randomly from the original sample. A $95 \%$ bootstrap lower confidence limit of the SB method for $S_{\mathrm{pk}}$ is constructed. If the calculated bootstrap lower confidence limit is found to be smaller than the specified 1.50 index value, we would judge that the process is incapable. Quality improvement activities will be initiated. Otherwise, the process is considered to be capable. From the 10000 bootstrap estimates $\hat{S}_{\mathrm{pk}}^{*}(i)$, the sample average can be calculated as

$\bar{S}_{\mathrm{pk}}^{*}=\frac{1}{10000} \sum_{i=1}^{10000} \hat{S}_{\mathrm{pk}}^{*}(i)$

and the sample standard deviation can be obtained as

$S_{\hat{S}_{\mathrm{pk}}}^{*}=\sqrt{\frac{1}{9999} \sum_{i=1}^{10000}\left(\hat{S}_{\mathrm{pk}}^{*}(i)-\bar{S}_{\mathrm{pk}}^{*}\right)^{2}}$.

Thus, the $95 \%$ SB lower confidence limit for $S_{\mathrm{pk}}$ can be constructed as

$\mathrm{LCB}=\bar{S}_{\mathrm{pk}}^{*}-Z_{0.05} \times S_{\hat{\mathrm{p}}_{\mathrm{kk}}}^{*}$.
The bootstrap resampling computation is rather complicated. In order to accelerate the computation, an integrated SAS computer program is developed (see Appendix A). The practitioners only need to input the manufacturing specifications, USL, LSL, a specified quality level of $S_{\mathrm{pk}}$ and a collected sample data of size $n$. The estimated value $\hat{S}_{\mathrm{pk}}$ and the SB lower confidence bound of $S_{\mathrm{pk}}$ may be easily obtained. Thus, whether or not the process is capable may be determined.

For the LCM real example we investigated, Table 2 shows the simulation result with $\hat{S}_{\mathrm{pk}}=1.72588$ using the SB bootstrap method, the lower confidence bound on $S_{\mathrm{pk}}$, was found to be 1.44244 , which is less than the specified 1.50 (the minimal value of the measure to reach a positive judgment). Thus, we may not conclude that the bonding precision of the bonding process is capable. Quality improvement team must immediately initiate some improvement activities to ensure the minimal requirement met.

\section{Conclusions}

Bootstrap resampling method is a nonparametric, computational intensive but effective estimation method, which is a data based simulation technique for statistical inference. In particular, one can use the nonparametric bootstrap method to estimate the sampling distribution of a statistic. Bootstrap method has been widely applied to statistical process control. In this paper, we apply the bootstrap method to the process yield measure $S_{\mathrm{pk}}$ to obtain the confidence bounds. The proposed approach makes it feasible for the engineers to perform approximate process performance testing using the calculated $S_{\mathrm{pk}}$. We also provide an efficient SAS computer program for the engineers to use. The program only requires an input of the manufacturing specification limits, and the sample data, then the estimated value $\hat{S}_{\mathrm{pk}}$ and SB lower confidence limit of $S_{\mathrm{pk}}$ will be outputted quickly. In summary, bootstrap lower confidence limit of $S_{\mathrm{pk}}$ may be used to evaluate process capability in statistical process control.

\section{Appendix A.}

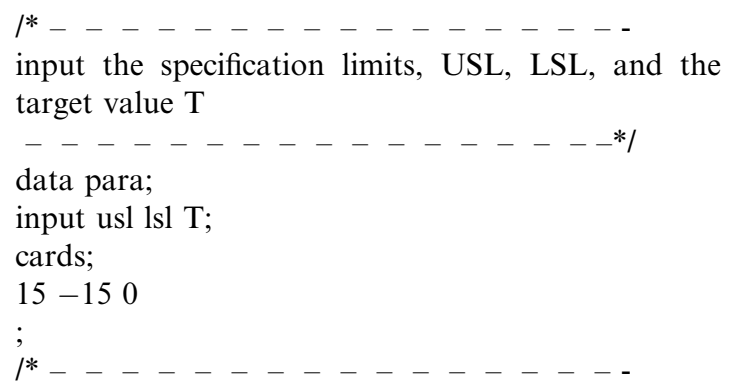


store the input of the original sample data of size $\mathrm{n}=64$

$---------------^{*} /$

data size;

set para;

array $x\{64\} \times 1-x 64$;

array $\mathrm{y}\{64\}$ y $1-\mathrm{y} 64$;

retain seed 123 ;

do $\mathrm{i}=1$ to 64 ;

inputx $\{\mathrm{i}\} @$;

end;

/* - - - - - - - - - - - - -

calculate the estimated $\mathrm{S}_{\mathrm{pk}}$ based on the original sample of $\mathrm{n}=64$

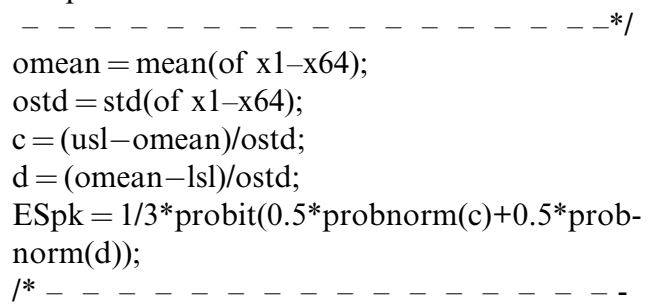

generate 10000 bootstrap samples from the original sample of $\mathrm{n}=64$

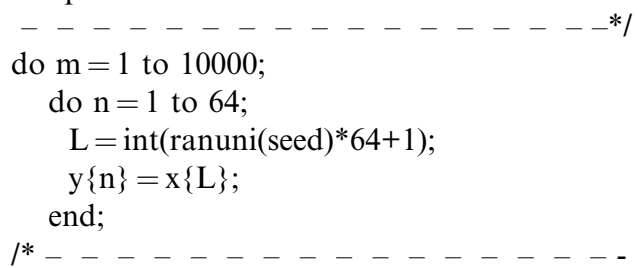

Compute the 10000 bootstrap estimates $\hat{\mathrm{S}}_{\mathrm{pk}}^{*}(i)$

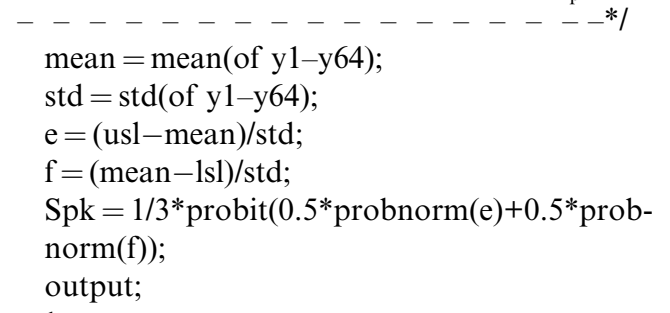

end;

/* $-\ldots-\ldots-\ldots-\ldots-\ldots$

Input the original sample data of $n=64$ sample observations

$----------------^{*} /$

cards;

$-0.984 .630 .78-1.672 .34$

$-1.97-0.07-2.322 .173 .78$

$0.35-1.203 .12-5.58-1.91$

$-1.18-0.66-3.165 .01-1.42$

$2.202 .202 .16-2.87-0.30$

$0.83-0.86-2.041 .245 .83$

$0.50-0.813 .07-1.42-2.42$

$-4.751 .02-6.501 .341 .06$

$0.105 .02-0.76-4.841 .28$
$2.190 .16-2.66-0.87-3.52$

$-0.051 .111 .470 .28-1.02$

$-9.24-2.01-2.304 .263 .41$

$3.342 .85-2.43-1.84$

;

/*_ $-\ldots-\ldots-\ldots-\ldots-\ldots-\ldots$

Compute $\overline{\mathrm{S}}_{\mathrm{pk}}^{*}$ and $\mathrm{S}_{\mathrm{S}_{\mathrm{pk}}}^{*}$ from the 10000 bootstrap estimates $\hat{\mathrm{S}}_{\mathrm{pk}}^{*}(i)$

$----------------^{*} /$

proc univariate data $=$ size normal plot;

var Spk;

output out $=$ out 1 mean $=$ mean

std = stddev;

/* _ - - - - - - - - - - - - - -

Calculate the lower confidence bound on the $\mathrm{S}_{\mathrm{pk}}$

$-\ldots-\ldots-\ldots-\ldots-\ldots-\ldots$

data sbcl;

set out1;

$\mathrm{SBLB}=$ mean-probit $(0.95)^{*}$ stddev;

proc print;

var SBLB;

run;

\section{References}

[1] Boyles RA. Process capability with asymmetric tolerances. Commun Statist: Simul Comput 1994;23(3):615-43.

[2] Lee JC, Hung HN, Pearn WL, Kueng TL. On the distribution of the estimated process yield index $S_{\mathrm{pk}}$. Qual Reliabil Eng Int, in press.

[3] Efron B. Bootstrap methods: another look at the Jackknife. Ann Statist 1979;7:1-26.

[4] Efron B. The Jackknife, the bootstrap and other resampling plans. Philadelphia, PA: Society for Industrial and Applied Mathematics; 1982.

[5] Efron B, Tibshirani RJ. Bootstrap methods for standard errors, confidence interval, and other measures of statistical accuracy. Statist Sci 1986;1:54-77.

[6] Efron B, Gong G. A leisurely look at the bootstrap, the Jackknife, and cross-validation. Am Statist 1983;37:36-48.

[7] Gunter B. Bootstrapping: how to make something from almost nothing and get statistically valid answers, Part I. Qual Progr 1991:97-103.

[8] Gunter B. Bootstrapping: how to make something from almost nothing and get statistically valid answers, Part III. Qual Progr 1992:119-22.

[9] Mooney CZ, Duval RD. Bootstrapping: a nonparametric approach to statistical inference. Newbury Park, CA: SAGE Publications; 1993.

[10] Young GA. Bootstrap: more than a stab in the dark? Statist Sci 1994;9:382-415.

[11] Franklin LA, Wasserman GS. Bootstrap lower confidence limits for capability indices. J Qual Technol 1992; 24(4): 196-210.

[12] Chou YM, Owen DB, Borrego SA. Lower confidence limits on process capability indices. J Qual Technol 1990; 22:223-9. 
[13] Bissell AF. How reliable is your capability index? Appl Statist 1990;39:331-40.

[14] Boyles RA. The Taguchi capability index. J Qual Technol 1991;23:17-26.
[15] Pearn WL, Chen JP. Bootstrap lower confidence bounds for process yield measure $S_{\mathrm{pk}}$. Working Paper, Department of Industrial Engineering and Management, National Chiao Tung University, Taiwan, 2001. 